\title{
Magnetic ordering and spin excitations in the frustrated magnet $\mathrm{MnSc}_{2} \mathrm{~S}_{4}$
}

\author{
A. Krimmel, ${ }^{1, *}$ M. Mücksch, ${ }^{2,3}$ V. Tsurkan, ${ }^{1,4}$ M. M. Koza, ${ }^{3}$ H. Mutka, ${ }^{3}$ C. Ritter, ${ }^{3}$ D. V. Sheptyakov, ${ }^{5}$ S. Horn, ${ }^{2}$ and \\ A. Loidl ${ }^{1}$ \\ ${ }^{1}$ Experimental Physics V, Center for Electronic Correlations and Magnetism, Augsburg University, D-86159 Augsburg, Germany \\ ${ }^{2}$ Experimental Physics II, Institute of Physics, Augsburg University, D-86159 Augsburg, Germany \\ ${ }^{3}$ Institut Laue Langevin, Boîte Postale 156 X, F-38042 Grenoble Cedex 9, France \\ ${ }^{4}$ Institute of Applied Physics, Academy of Sciences of Moldova, MD2028, Chisinau, Republic of Moldova \\ ${ }^{5}$ Laboratory for Neutron Scattering, ETH Zürich \& PSI Villigen, CH-5232 Villigen, PSI, Switzerland \\ (Received 31 August 2005; revised manuscript received 30 November 2005; published 18 January 2006)
}

\begin{abstract}
The $A$-site spinel compound $\mathrm{MnSc}_{2} \mathrm{~S}_{4}$ exhibits strong frustration effects with a low ordering temperature $\left(T_{N}=2.3 \mathrm{~K}\right)$. Detailed neutron-scattering investigations have been performed to study the magnetic ordering process and the associated magnetic excitations. The magnetic structure found at $T=1.5 \mathrm{~K}$ is a spiral within the $a-b$ plane characterized by a propagation vector of $\mathbf{q}=(3 / 4,3 / 4,0)$. The magnetic ordering process is spectroscopically reflected by a change from a purely quasielastic to an inelastic response. The quasielastic line width corresponds to a magnetic energy scale of $\approx 1 \mathrm{meV}$ which is consistent with the spin wave stiffness as determined from the magnon dispersion at low temperatures.
\end{abstract}

DOI: $10.1103 /$ PhysRevB.73.014413

PACS number(s): 75.40.Gb, 78.70.Nx, 75.30.Et, 67.90.+z

\section{INTRODUCTION}

The cubic spinel structure is one of the most frequently encountered structure types among transition metal compounds of stoichiometry $A B_{2} X_{4}$. The spinel structure is described by space group $F d \overline{3} m$ with tetrahedrally coordinated $A$ sites and octahedrally coordinated $B$ sites. It is well known that the $B$-site ions form a pyrochlore lattice with inherent strong geometric frustration (GF) for nearest-neighbor antiferromagnetic (AFM) interactions. GF denotes the inability of a system to satisfy all pairwise interactions. ${ }^{1}$ In spin systems frustration suppresses long-range magnetic order and strongly enhances spin fluctuations. The ratio of the CurieWeiss temperature reflecting the energy scale of the dominating magnetic exchange to the ordering temperature is commonly taken as a measure of frustration. ${ }^{1}$ However, at sufficiently low temperatures, any additional interaction other than the leading exchange (e.g., next-nearest-neighbor or dipolar interactions) enters in a nonperturbative way usually yielding to an ordered ground state. Consequently, a large variety of different fascinating ground states are possible for frustrated systems, ${ }^{1}$ which depend on the very details of the interactions involved (e.g., exchange, anisotropy, magnitude of the spin, etc.). Spin liquids, ${ }^{2}$ spin-ice states, ${ }^{3,4}$ clusters or loops of a finite number of spins, ${ }^{5-7}$ heavyfermion-like behavior, ${ }^{8}$ as well as singlet formation ${ }^{9}$ are experimentally observed or theoretically calculated. In some cases such as $\mathrm{ZnV}_{2} \mathrm{O}_{4}$, magnetic frustration is lifted via a coupling between spin and lattice degrees of freedom, ${ }^{10}$ resulting in a magnetic ${ }^{11}$ or spin-driven ${ }^{12}$ Jahn-Teller effect.

Much less attention has been payed to the $A$-site lattice of spinel compounds. Investigations of normal $A B_{2} X_{4}$ spinels with only $A-A$ magnetic interactions are rare and only a few examples of long-range magnetic and orbital order are known. Very recently the existence of strong GF has been demonstrated for a number of spinel compounds, not only for the spin sector but also for the orbital and structural de- grees of freedom resulting in unusual ground states such as a spin-orbital liquid, ${ }^{13}$ an orbital glass, ${ }^{14}$ or strong magnetodielectric effects. ${ }^{15}$ For $\mathrm{FeSc}_{2} \mathrm{~S}_{4}$ the absence of any long-range magnetic order above $50 \mathrm{mK}$ combined with the high CurieWeiss temperature of $-45 \mathrm{~K}$ gives rise to one of the largest frustration parameters $(\geqslant 1000)$ ever observed ${ }^{16}$ and evidences the presence of strong frustration effects in magnetic $A$-site spinels. ${ }^{17}$

Roth ${ }^{18}$ discussed the principal superexchange paths linking the $A$ sites in the normal spinel. A schematic view of the spinel structure with frame of reference along the principal crystallographic axis and the $A$-site magnetic exchange of $\mathrm{MnSc}_{2} \mathrm{~S}_{4}$ corresponding to a projection onto the [110]-[111] plane is shown in Fig. 1. All superexchange paths involve at least three intermediate ions and therefore are certainly weak. The exchange $J$ between four nearest neighbors (NN) has a sixfold interaction path and the exchange $J^{\prime}$ between twelve next-nearest neighbors (NNN) exhibits a twofold in-
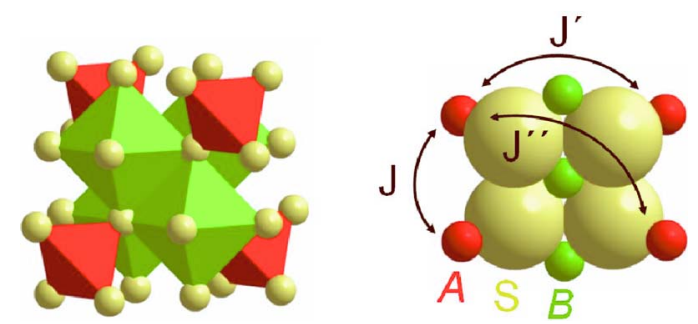

FIG. 1. (Color online) Coordination polyhedra of the spinel structure of $\mathrm{MnSc}_{2} \mathrm{~S}_{4}$. A-site $\mathrm{Mn}$ (red, dark grey) is embedded in a tetrahedral environment of sulphur (yellow, light grey), while $B$-site $\mathrm{Sc}$ has octahedral environment (left part). The $A$-site exchange is mediated via three different Mn-S-Sc-S-Mn interaction paths. The configuration containing nearest, next-nearest, and next-nextnearest neighbors of the $A$-site sublattice with corresponding exchange constants $J, J^{\prime}$, and $J^{\prime \prime}$ is shown at the right. The right part corresponds to a projection of four neighboring $A$-site ions onto the [110]-[111] plane. 
teraction path, resulting in a multiplicity for $J$ and $J^{\prime}$ of 24 each. The exchange paths $J^{\prime \prime}$ to the twelve next-next-nearest neighbors (NNNN) are single fold. It should be stressed that although NN, NNN, and NNNN shells exhibit different spacings in the crystal lattice, the lengths of the exchange paths are rather similar. Therefore, the weak indirect interaction is strengthened by the large multiplicity of the exchange paths. Though the frustration effects in the network shown in Fig. 1 are not obvious, it should be noted that the $A$-site ions form a diamond lattice, consisting of two interpenetrating fcc sublattices. Face centered cubic (fcc) lattices which are coupled antiferromagnetically are highly frustrated. In addition, the coordination shells form highly frustrated networks: the NNs of the $A$-site sublattice form tetrahedrons, the NNN polyhedron is formed by triangles and squares and the NNNN shell forms a polyhedron of triangles and hexagons, that can be mapped onto a Kagomé lattice which is a classical example of a frustrated lattice.

$\mathrm{MnSc}_{2} \mathrm{~S}_{4}$ crystallizes in the normal cubic spinel structure. The $B$ site is occupied by nonmagnetic Sc ions and the $A$ site by magnetic $\mathrm{Mn}^{2+}$ ions revealing a half-filled $d$ shell with $S=5 / 2$ and zero orbital momentum. ${ }^{19}$ The tetrahedral crystal field splits the $3 d$ electron manifold into a lower doublet $(e)$ and an excited triplet $\left(t_{2}\right)$ state. At elevated temperatures, the magnetic susceptibility of $\mathrm{MnSc}_{2} \mathrm{~S}_{4}$ follows a Curie-Weiss law with a Curie-Weiss temperature of $\theta_{\mathrm{CW}}=-22.9 \mathrm{~K} .{ }^{16} \mathrm{At}$ $T=2.3$ and $1.9 \mathrm{~K}$, anomalies of the susceptibility, as well as a corresponding double peak structure in the specific heat signal the onset of long-range magnetic order. The ratio of the Curie-Weiss temperature to the ordering temperature $\theta_{\mathrm{CW}} / T_{N} \approx 10$ indicates significant frustration effects. Between $T_{N} \approx 2 \mathrm{~K}$ and $-\theta_{\mathrm{CW}} \approx 23 \mathrm{~K} \mathrm{MnSc} \mathrm{S}_{4}$ has been characterized as a spin liquid. ${ }^{16}$ Here we report on a detailed neutron scattering study on $\mathrm{MnSc}_{2} \mathrm{~S}_{4}$ aimed to elucidate the magnetic ordering process and its associated magnetic excitations.

\section{EXPERIMENTAL RESULTS AND DISCUSSION}

\section{A. Sample preparation and characterization}

Polycrystalline samples of $\mathrm{MnSc}_{2} \mathrm{~S}_{4}$ were prepared by solid-state reactions of stoichiometric mixtures of the highpurity elements at $900{ }^{\circ} \mathrm{C}$. In order to achieve single phase material and to obtain good homogeneity, the sintering process was repeated several times with subsequent regrinding, pressing and firing. Characterization by conventional x-ray powder diffraction using $\mathrm{Cu} K_{\alpha 1}$ radiation confirmed the normal cubic spinel structure without indications of parasitic phases. A Rietveld refinement of the diffraction patterns resulted in a lattice constant of $a=10.621(7) \AA$ and a sulphur fractional coordinate $x=0.257(1)$ at room temperature consistent with Ref. 16.

The temperature dependence of the magnetic susceptibility has been measured in a SQUID magnetometer for 1.8 $\leqslant K \leqslant 400 \mathrm{~K}$. As reported previously, ${ }^{16}$ anomalies in the temperature dependencies of the magnetic susceptibility and in the heat capacity signal the onset of long-range magnetic order. As shown in detail in the inset of Fig. 2, a maximum

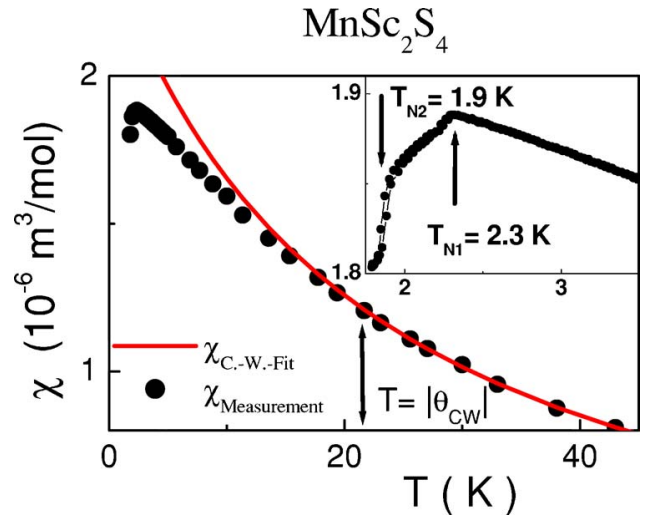

FIG. 2. (Color online) Low-temperature part of the magnetic susceptibility of $\mathrm{MnSc}_{2} \mathrm{~S}_{4}$ and the corresponding Curie-Weiss fit as determined for temperatures between 50 and $400 \mathrm{~K}$ (full line). $\theta_{\mathrm{CW}}$ and the ordering temperatures $T_{N 1}$ and $T_{N 2}$ are indicated.

of the susceptibility is reached at $T_{N} \equiv T_{N 1}=2.3 \mathrm{~K}$ and a sudden fall off occurs at $T_{N 2}=1.9 \mathrm{~K}$. At elevated temperatures in the paramagnetic regime a Curie-Weiss behavior is observed with a Curie-Weiss temperature $\theta_{\mathrm{CW}}=-22.9 \mathrm{~K}$ and an effective paramagnetic moment of $\mu_{\mathrm{eff}}^{\mathrm{para}}=5.8 \mu_{B}$ as expected for $\mathrm{Mn}^{2+}$ in a high-spin configuration. Figure 2 shows the lowtemperature part $(T<45 \mathrm{~K})$ of the magnetic susceptibility of $\mathrm{MnSc}_{2} \mathrm{~S}_{4}$ with a Curie-Weiss fit for which only data for 50 $\leqslant T \leqslant 400 \mathrm{~K}$ have been taken into account. The figure evidences clear deviations from a Curie-Weiss behavior below $23 \mathrm{~K}$, thus pointing towards the evolution of short-range magnetic correlations for $T<\left|\theta_{\mathrm{CW}}\right|$. Hence, $\mathrm{MnSc}_{2} \mathrm{~S}_{4}$ exhibits a large temperature range between $T_{N 1}=2.3 \mathrm{~K}$ and $\left|\theta_{\mathrm{CW}}\right|$ $=23 \mathrm{~K}$ which is characterized by a dynamic short-ranged ordered state due to frustration effects.

\section{B. Neutron powder diffraction}

Neutron powder diffraction experiments were performed on the instruments D20 at the Institut Laue Langevin, Grenoble (France) and on HRPT at the Paul Scherrer Institut, Villigen (Switzerland). Different incident neutron wavelengths of 1.154, 1.886, and $2.45 \AA$ have been employed. For the ease of comparing different measurements, all diffraction patterns have been transformed into a function of momentum transfer $Q$ in absolute units $\AA^{-1}$. Powdered samples were filled in a vanadium container and mounted in conventional cryostats allowing for temperatures $1.5 \leqslant T \leqslant 300 \mathrm{~K}$. The diffraction data have been analyzed by standard Rietveld refinement employing the FULLPROF program..$^{20}$

The crystal structure of $\mathrm{MnSc}_{2} \mathrm{~S}_{4}$ is most accurately determined by measurements employing neutrons of $\lambda$ $=1.154 \AA$ corresponding to diffraction intensities for wave vector transfers up to $Q=10.8 \AA^{-1}$. This wave length has been used for measurements at $T=1.5,25$, and $50 \mathrm{~K}$, respectively. Figure 3 shows the corresponding diffraction pattern of $\mathrm{MnSc}_{2} \mathrm{~S}_{4}$ in the paramagnetic regime at $T=25 \mathrm{~K}$. For the crystallographic structure, the following parameters have been refined: scale factor, zero point, four background parameters, three resolution parameters, the lattice parameter $a$, the sulfur position at $(x, x, x)$, and isotropic temperature fac- 


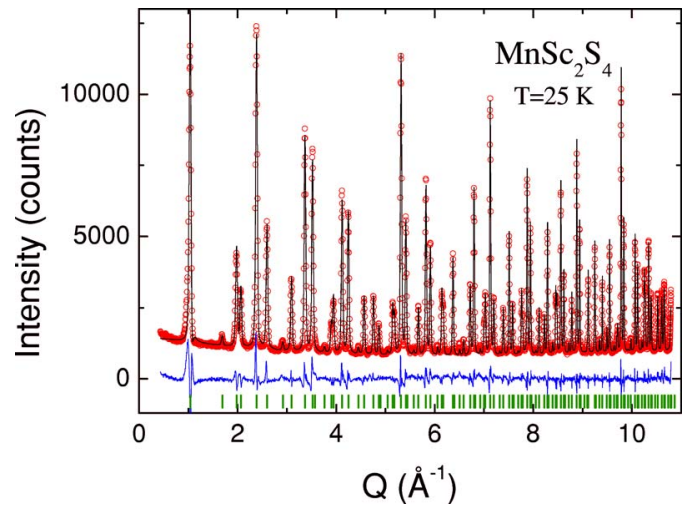

FIG. 3. (Color online) Neutron powder diffraction pattern of $\mathrm{MnSc}_{2} \mathrm{~S}_{4}$ at $T=25 \mathrm{~K}$ measured on HRPT $(\lambda=1.154 \AA)$. Shown are observed (open circles) and calculated (full line) intensities, as well as their difference (full line at the bottom) and the corresponding peak positions.

tors for $\mathrm{Mn}, \mathrm{Sc}$, and $\mathrm{S}$, respectively. The normal cubic spinel structure has been confirmed for the entire investigated temperature range. The results of the refined crystal structure of $\mathrm{MnSc}_{2} \mathrm{~S}_{4}$ are summarized in Table I. Table I shows that the sulphur positional parameter is very close to the value of 0.25 of the ideal spinel structure and that the isotropic temperature factor of Sc is rather high.

At low temperatures $T \leqslant T_{N}$, additional magnetic reflections show up between the nuclear Bragg peaks thus reflecting antiferromagnetic order. Due to the neutron magnetic form factor, magnetic intensities are weakened for high momentum transfers. Therefore, diffraction data with $\lambda$ $=1.886 \AA$ (on HRPT) and $2.45 \AA$ (on D20), respectively, have been used to determine the magnetic structure of $\mathrm{MnSc}_{2} \mathrm{~S}_{4}$. The positions of all magnetic Bragg reflections can be indexed by $h k l_{\text {nuc }} \pm \mathbf{q}$ with a propagation vector $\mathbf{q}$ $=(0.75,0.75,0)$. In real space this propagation vector corresponds to a magnetic unit cell of $4 a / 3,4 b / 3, c$, i.e., three magnetic periods are formed within a fourfold crystallographic unit cell. Since the magnetic structure rotates in the $x$ and $y$ directions three times in a fourfold cell for eight atoms, the phase in the $x$ (or $y$ ) direction is $3 / 8$. Perpendicular to $\mathbf{q}$ are magnetic Mn layers with a distance of $d=1 / 4 \sqrt{\left(a^{2}+b^{2}\right)}$ and therefore there are 16 layers in a fourfold cell giving rise to 6 magnetic rotations along the (110) direction. The magnetic structure of $\mathrm{MnSc}_{2} \mathrm{~S}_{4}$ is a spiral with magnetic moments confined within the $a-b$ plane. The refined diffraction

TABLE I. Crystal structure of $\mathrm{MnSc}_{2} \mathrm{~S}_{4}$ as resulting from the Rietveld refinements within space group $F d \overline{3} m$. Listed are the lattice parameter $a$, sulphur positional parameter $x$ in fractional coordinates, isotropic temperature factors for $\mathrm{Mn}, \mathrm{Sc}$, and $\mathrm{S}$, respectively, and the residuals $R_{\mathrm{Bragg}}$.

\begin{tabular}{lllll}
\hline \hline$T(\mathrm{~K})$ & $a(\AA)$ & $x(\mathrm{~S})$ & $B_{\text {iso }}(\mathrm{Mn}, \mathrm{Sc}, \mathrm{S})$ & $R_{\text {Bragg }}$ \\
\hline 1.6 & $10.5989(1)$ & $0.2577(2)$ & $0.11(3), 0.21(1), 0.13(4)$ & 0.041 \\
25 & $10.5995(1)$ & $0.2577(1)$ & $0.09(2), 0.21(1), 0.12(2)$ & 0.035 \\
50 & $10.5992(1)$ & $0.2576(1)$ & $0.11(2), 0.23(1), 0.12(3)$ & 0.039 \\
\hline \hline
\end{tabular}

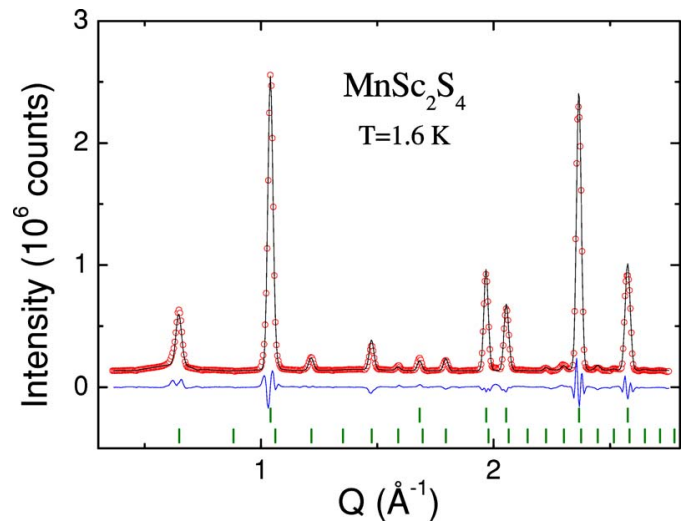

FIG. 4. (Color online) Detailed neutron-powder-diffraction pattern of $\mathrm{MnSc}_{2} \mathrm{~S}_{4}$ at $T=1.6 \mathrm{~K}$ in the low- $Q$ range $(\lambda=2.45 \AA)$ measured on D20. Shown are observed (open circles) and calculated intensities (full line), as well as their difference (bottom line). The vertical bars indicate the peak positions corresponding to the crystallographic (upper row) and magnetic (lower row) structure, respectively.

pattern at $T=1.6 \mathrm{~K}$ is shown in Fig. 4 and the corresponding magnetic structure is shown in Fig. 5. The magnetic structure is consistent with recent NMR investigations ${ }^{21}$ of $\mathrm{MnSc}_{2} \mathrm{~S}_{4}$. It is important to note that the symmetry of the magnetic structure is incompatible with the underlying cubic spinel type crystal structure. Most probably, the crystal symmetry is actually reduced but below the resolution of the present neutron powder diffraction experiments. This scenario would be an alternative explanation of the two subsequent phase transitions at about $2 \mathrm{~K}$ which were unambiguously identified via heat capacity experiments. Here one transition could be of magnetic and one of structural origin driven by a strong coupling of spin and lattice degrees of freedom, theoretically described by spin-driven Jahn-Teller transitions. ${ }^{11,12}$ However, certainly more experimental work is needed to clarify the situation. The symmetry reduction may occur via a small distortion that leads to a change of the crystal metric. In such

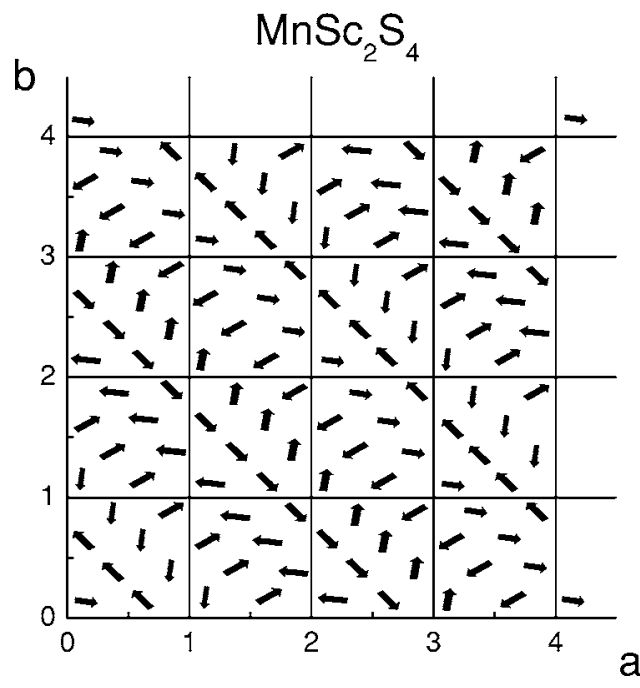

FIG. 5. The magnetic structure of $\mathrm{MnSc}_{2} \mathrm{~S}_{4}$ within the $a-b$ plane showing a cycloidic spin arrangement. 


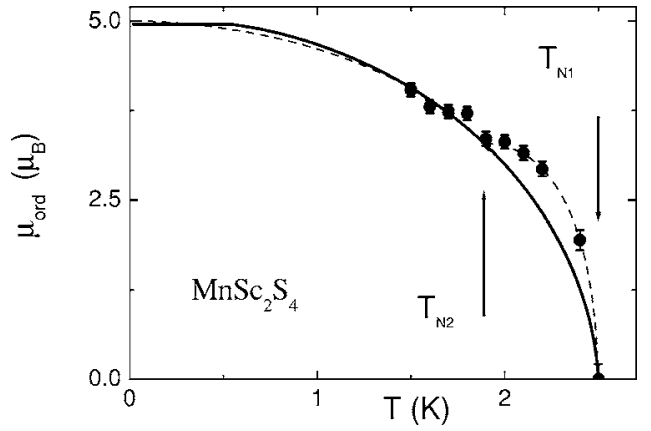

FIG. 6. Temperature dependence of the ordered magnetic moment of $\mathrm{MnSc}_{2} \mathrm{~S}_{4}$ compared to an $S=5 / 2$ Brillouin function (full line). The dashed line is a guide to the eye according to two magnetic transitions occurring at $T_{N 1}$ and $T_{N 2}$, respectively, as indicated by the arrows.

a case, ultrahigh resolution synchrotron diffraction experiments may reveal a structural change associated with the magnetic ordering transition in $\mathrm{MnSc}_{2} \mathrm{~S}_{4}$, though the necessary low temperatures certainly pose a problem. However, a symmetry reduction may also take place via a structural modulation that preserves the cubic metric of the system. In this case, diffraction experiments probing the global structure may be inappropriate as it may differ from the local structure.

The ordered magnetic moment of $\mathrm{MnSc}_{2} \mathrm{~S}_{4}$ amounts $4.05(5) \mu_{B}$ at $T=1.5 \mathrm{~K}$ which is significantly reduced compared to $5 \mu_{B}$ expected for $\mathrm{Mn}^{2+}$ with $S=5 / 2$ and $g=2$. Due to the low ordering temperature of $T_{N 1}=2.3 \mathrm{~K}$ the lowest experimentally accessible temperature of $1.5 \mathrm{~K}$ is insufficient to reach saturation. The diffraction measurements at various temperatures result in a temperature dependent ordered magnetic moment that roughly follows a simple mean field behavior. The experimental data and a corresponding fit according to a $S=5 / 2$ Brillouin function are shown in Fig. 6 . Interestingly, the deviations of the temperature-dependent ordered magnetic moment from mean field behavior may reflect the twofold magnetic transition at $T_{N 1}$ and $T_{N 2}$, respectively, as indicated by the dashed line and the arrows in Fig. 6.

The strongest magnetic intensity of $\mathrm{MnSc}_{2} \mathrm{~S}_{4}$ corresponding to the first magnetic reflection $( \pm 3 / 4, \pm 3 / 4,0)$ is found around $0.62 \AA^{-1}$. The detailed shape of this reflection for various temperatures is shown in the upper part of Fig. 7. It consists of two different components; a sharp resolution limited Gaussian line and an additional broad Lorentzian shaped scattering contribution. The temperature dependence of these two magnetic intensities is shown in the lower frame of Fig. 7. The narrow Gaussian Bragg peak vanishes around $2.3 \mathrm{~K}$ thus coinciding with the upper anomaly of the magnetic susceptibility and specific heat. It is therefore a signature of long range magnetic order of $\mathrm{MnSc}_{2} \mathrm{~S}_{4}$ which is established below $T_{N 1}=2.3 \mathrm{~K}$. On the other hand, the second, intrinsically broadened magnetic intensity extends to much higher temperatures and can be traced back to $23 \mathrm{~K}$ corresponding to $\theta_{\mathrm{CW}}$. These results confirm that short-range magnetic order develops below $\theta_{\mathrm{CW}}$ but that long-range magnetic order is suppressed down to $T_{N} \approx \theta_{\mathrm{CW}} / 10$ due to frustration effects

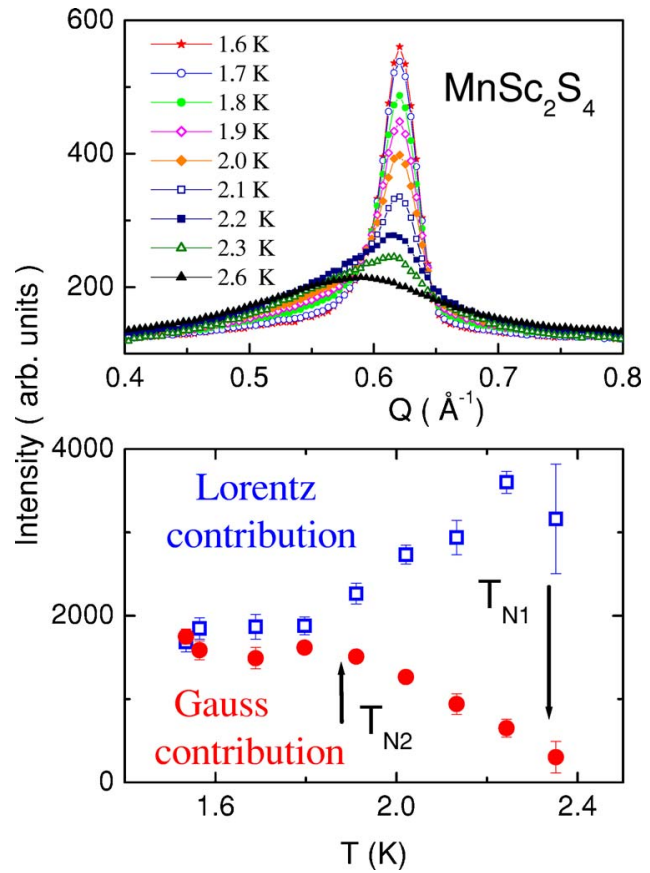

FIG. 7. (Color online) Upper frame: The temperature dependence of the first magnetic Bragg reflection of $\mathrm{MnSc}_{2} \mathrm{~S}_{4}$ around $0.62 \AA^{-1}$ measured on D20. The magnetic signal has two components: a sharp resolution limited and Gaussian shaped component and an additional broad Lorentzian shaped line. Lower frame: Temperature dependence of the corresponding two magnetic intensities based on fit results of HRPT data. Magnetic transition temperatures are indicated by arrows.

and that even at $T=1.5 \mathrm{~K}$ short-range fluctuations seem still to persist. More information about these different magnetic contributions can be extracted from neutron spectroscopic measurements, as will be detailed in the next paragraph.

\section{Neutron spectroscopy}

Inelastic neutron scattering experiments were performed in a temperature range $1.6<T<300 \mathrm{~K}$ on the time-of-flight spectrometer IN6 at the Institut Laue Langevin, Grenoble. Most experiments have been carried out using an incident neutron wave length of $5.1 \AA$ A. For an increased resolution, a few measurements have also been performed with neutrons of $5.9 \AA$. Additionally, an empty can and a vanadium standard have been measured to account for background and detector efficiency, respectively. The raw data have been corrected in a standard way employing the LAMP program package $^{22}$ with a conversion of time-of-flight to energy transfer and a constant $Q$ or constant energy mapping resulting finally in $S(Q, \omega)$. The dynamic structure factor $S(Q, \omega, T)$ is directly proportional to the imaginary part of the generalized susceptibility

$$
S(Q, \omega, T)=\left(1-e^{-h \omega / k_{B} T}\right)^{-1} \chi^{\prime \prime}(Q, \omega, T) .
$$

The first term represents the detailed balance factor accounting for thermal population. An exponential spin relaxation results in a Lorentzian shaped quasielastic line (multiplied 


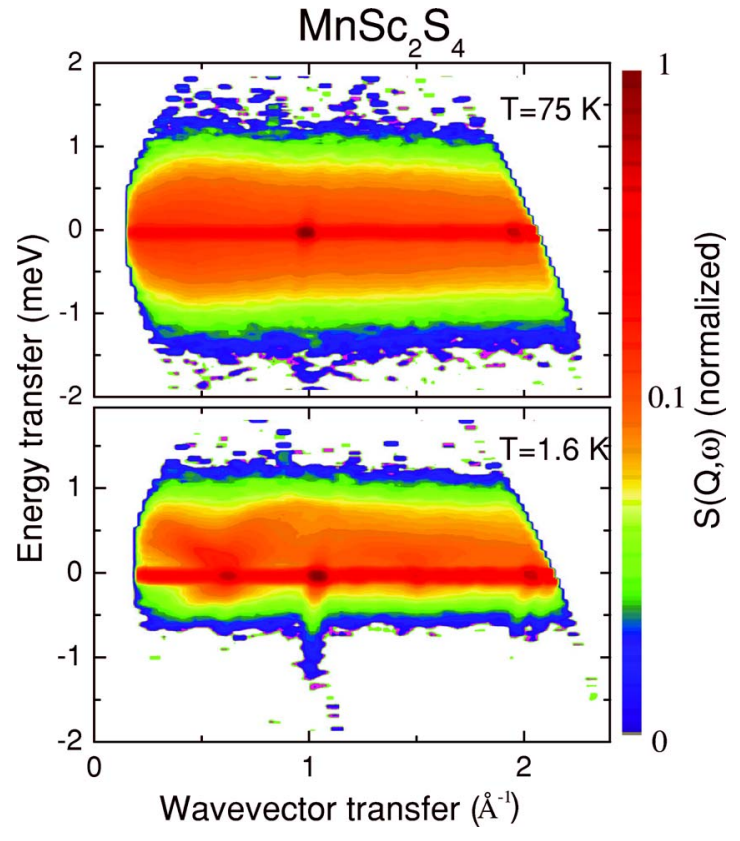

FIG. 8. (Color online) Contour plot of $S(Q, \omega)$ of $\mathrm{MnSc}_{2} \mathrm{~S}_{4}$ at $T=75 \mathrm{~K}$ in the paramagnetic state (upper panel) and at $T=1.6 \mathrm{~K}$ in the afm magnetically ordered state (lower panel), respectively. Magnetic intensities are shown in a suitable color coding on a logarithmic scale.

with the detailed balance factor and convoluted with the instrumental resolution)

$$
\chi^{\prime \prime}(Q, \omega, T)=\omega \Gamma(Q, T) \frac{\chi_{0}(Q, T)}{\omega^{2}+\Gamma^{2}(Q, T)} .
$$

$\Gamma(Q)$ is the $Q$-dependent full width at half maximum (FWHM) and $\chi_{0}(Q, T)$ is related to the bulk susceptibility $\chi_{0}(T)$ via the neutron magnetic form factor $f(Q)$ by $\chi_{0}(Q, T)=f^{2}(Q) \chi_{0}(T)$. The quasielastic linewidth $\Gamma(Q, T)$ corresponds to the characteristic energy scale of the system. Figure 8 shows a contour map of $S(Q, \omega)$ at $T=75 \mathrm{~K}$ in the paramagnetic state (upper panel) and at $T=1.6 \mathrm{~K}$ in the magnetically ordered state (lower panel), respectively. Strong intensities in form of a resolution limited (FWHM $\approx 81.1 \mu \mathrm{eV}$ ) narrow riff around zero energy transfer appear due to incoherent scattering contributions. Dark spots at $Q$ values of around 1 and $2 \AA^{-1}$ are identified with the allowed nuclear Bragg reflections (111) and (311), respectively. The (220) reflection at $1.7 \AA^{-1}$ is not clearly seen. At $T=75 \mathrm{~K}$, roughly $Q$-independent additional quasielastic scattering intensity extends up to $\approx 1 \mathrm{meV}$ energy transfer (see Fig. 8). As evident from Fig. 8, $\Gamma \approx 1 \mathrm{meV}$ thus represents the characteristic energy scale of spin fluctuations which corresponds to approximately one half of the Curie Weiss temperature $\theta_{\mathrm{CW}}=-22.9 \mathrm{~K}$. At $T=1.6 \mathrm{~K}$, in the magnetically ordered state, additional magnetic Bragg peaks are observed as dark spots, most pronounced around 0.6 and $1.5 \AA^{-1}$, respectively. Rather well defined magnon excitation branches originate from these magnetic Bragg peaks as they represent the center of the magnetic Brillouin zone.

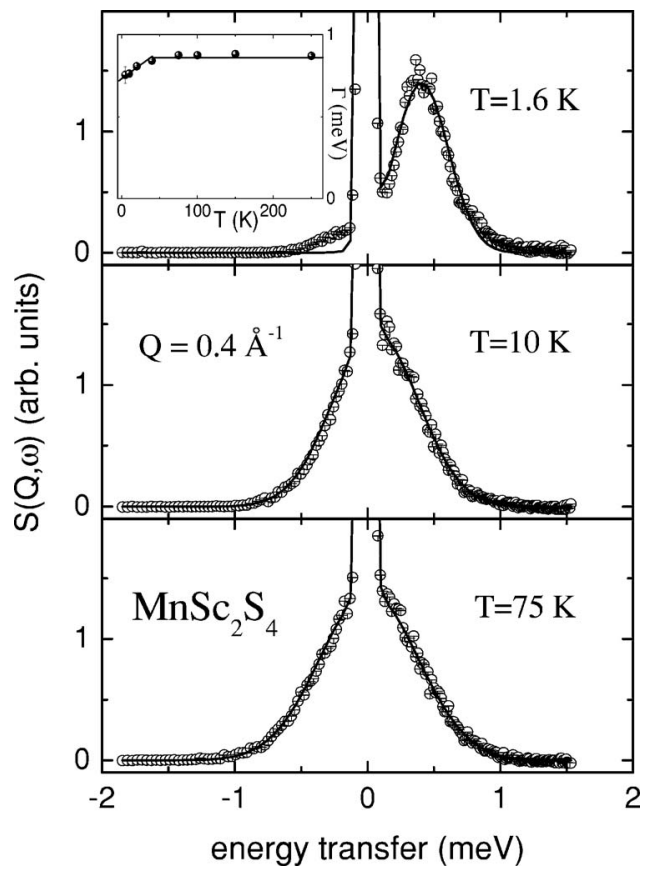

FIG. 9. $S(\omega)$ at constant $Q=0.4 \AA^{-1}$ of $\mathrm{MnSc}_{2} \mathrm{~S}_{4}$ at $T=75 \mathrm{~K}$ (lower panel), $T=10 \mathrm{~K}$ (middle), and at $T=1.6 \mathrm{~K}$ (upper panel) in the afm magnetically ordered state, respectively. The inset shows the temperature dependence of the quasielatic line width.

The evolution from a quasielastic response in the paramagnetic regime to inelastic excitations in the magnetically ordered state is also illustrated in Fig. 9, where $S(\omega)$ is shown for constant $Q=0.4 \AA^{-1}$ at various temperatures. Due to neutron kinematics, $0.4 \AA^{-1}$ corresponds to the lowest accessible $Q$ value where nonmagnetic scattering is negligible. Moreover, as evident from Fig. 8, $Q=0.4 \AA^{-1}$ is close to the Brillouin zone boundary of the magnetic unit cell. The magnetic intensities have been fitted (solid lines in Fig. 9) according to a single Lorentzian line using Eqs. (1) and (2).

The inset of Fig. 9 shows the temperature dependence of the quasielastic linewidth at $Q=0.4 \AA^{-1}$. Above $T \geqslant 40 \mathrm{~K}$, $\Gamma(T)$ remains constant at $\Gamma=0.87 \mathrm{meV}$ at least up to $250 \mathrm{~K}$ $(=21.55 \mathrm{meV})$. This constant line width indicates that no excited states are available with the given thermal energy, corresponding to a large gap of at least $22 \mathrm{meV}$. On cooling below $40 \mathrm{~K}$, the line width decreases only slightly and extrapolates to a finite value of $\Gamma(T \rightarrow 0)=0.72 \mathrm{meV}$. For thermally activated fluctuations, the quasielastic line width follows a linear Korringa law. The rather constant value of $\Gamma(T)$ for $\mathrm{MnSc}_{2} \mathrm{~S}_{4}$ shows that magnetic fluctuations do not freeze out continuously upon cooling due to frustration effects. This is also evidenced from Fig. 8 which shows that the magnetic intensities become significantly modulated in $Q$ at low temperatures, but the overall energy scale of magnetic fluctuations is obviously almost identical for $T=75$ and $1.6 \mathrm{~K}$.

The magnetic scattering of $\mathrm{MnSc}_{2} \mathrm{~S}_{4}$ at low temperature $(T=1.6 \mathrm{~K})$ within the long-range ordered magnetic state shows a well defined spin wave dispersion. Due to the directional averaging in powder experiments, such a dispersion is visible only for weak anisotropy as it is expected for a pure spin system in a high symmetry crystal structure. The dy- 


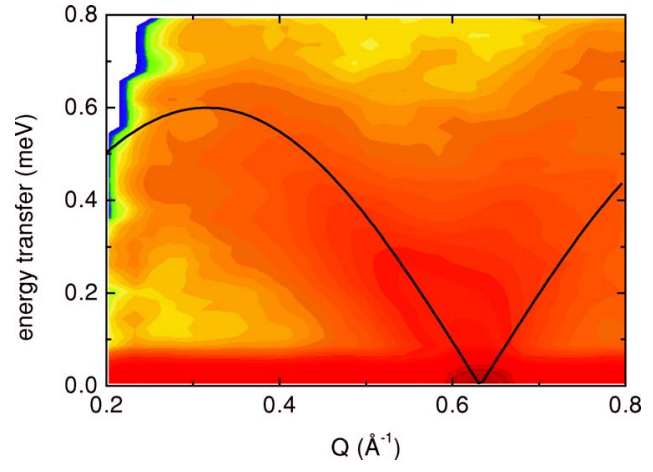

FIG. 10. (Color online) Detailed part of $S(Q, \omega)$ around the first magnetic reflection. The full line corresponds to a fit of the magnetic excitations according to an antiferromagnetic spin wave dispersion.

namic magnetic structure factor of $\mathrm{MnSc}_{2} \mathrm{~S}_{4}$ around the first magnetic Bragg reflection corresponding to the zone center of the first magnetic Brillouin zone is shown in Fig. 10 on an expanded scale. The magnon branches starting from the zone center are clearly visible. The black lines correspond to a fit to the data according to an antiferromagnetic spin wave dispersion relation $\hbar \omega=z S J \sqrt{\sin ^{2}(q a)}$ with $S=5 / 2$ being the spin value, $z=24$ the multiplicity of the NN magnetic exchange and $J$ the effective magnetic exchange coupling, respectively.

The inelastic neutron scattering data also provide information on the origin of the additional broad diffraction intensity around the first magnetic Bragg reflection. This is documented in Fig. 11. In the upper part of this figure, a section of the diffraction profile corresponding to a strip of $S(Q, \omega)$ along zero energy transfer $(\hbar \omega=0)$ is shown for various energy width $(0.1 \leqslant \Delta \hbar \omega \leqslant 2.0 \mathrm{meV})$ around the first magnetic Bragg reflection at $T=1.6 \mathrm{~K}$. As evident from the figure, a broad additional component in the diffraction pattern emerges upon increasing energy width which, however, corresponds to inelastic scattering processes due to magnetic excitations. The broad diffraction component can be monitored far above the magnetic ordering temperature up to the Curie-Weiss temperature of $\theta_{\mathrm{CW}} \approx 23 \mathrm{~K}$. This result implies that strong magnetic correlations extent up to $T \approx \theta_{\mathrm{CW}}$. The frustrated magnet $\mathrm{MnSc}_{2} \mathrm{~S}_{4}$ therefore shows a transition from a high-temperature paramagnet $\left(T \geqslant \theta_{\mathrm{CW}}\right)$ via a spin liquid phase for intermediate temperatures $\left(T_{N} \leqslant T \leqslant \theta_{\mathrm{CW}}\right)$ to a magnetically long-range ordered spin ground state for low temperatures $\left(T \leqslant T_{N}\right)$. We now consider pure elastic scattering processes extracted by a constant energy cut of $S(Q, \omega)$ at $\hbar \omega=0$ with a very narrow energy width of $\hbar \omega=50 \mu \mathrm{eV}$ corresponding to the instrumental resolution for an incident neutron wavelength of $\lambda=5.9 \AA$. Such elastic scattering intensities are shown in the lower part of Fig. 11 for various temperatures. Clearly visible, a double peak structure exists in a small temperature region above $T_{N 1}$. The double peak structure and its slightly reduced $Q$ position, as compared to the $Q$ position of the first magnetic reflection $(3 / 4,3 / 4,0)$ at $T=1.5 \mathrm{~K}$ can be accounted for by a propagation vector with likewise reduced components. The small deviation from the value of 3/4 indicates an incommensurate magnetic modula-

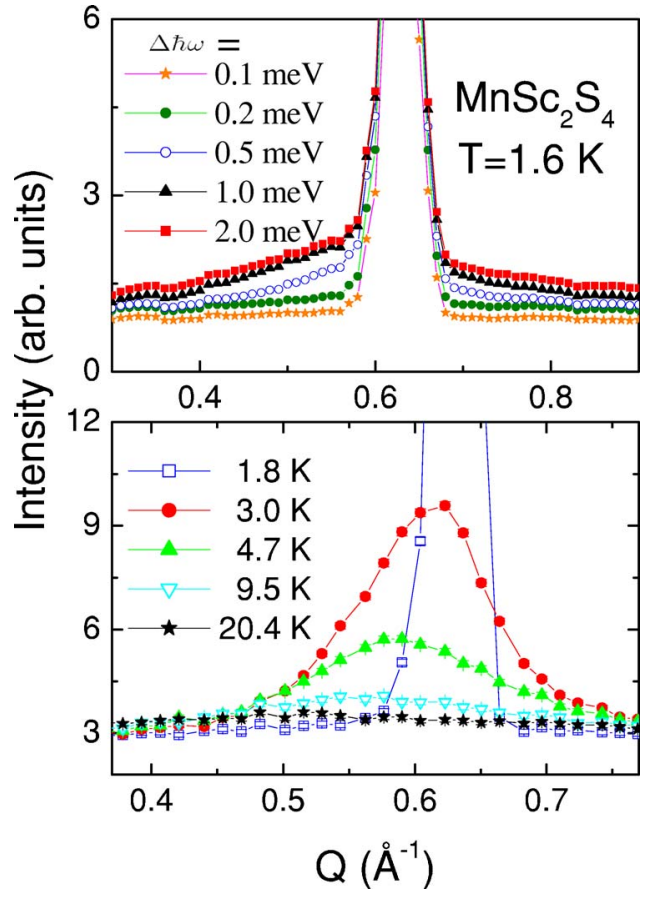

FIG. 11. (Color online) Upper part: Energy integrated structure factor $S(Q)$ for various energy width to illustrate the role of magnetic fluctuations onto the peak shape. Lower part: Pure elastic scattering of $\mathrm{MnSc}_{2} \mathrm{~S}_{4}$ for various temperatures.

tion. Therefore, the magnetic ordering process of $\mathrm{MnSc}_{2} \mathrm{~S}_{4}$ may be described as follows: On cooling, magnetic correlations develop below $T=\Theta_{\mathrm{CW}} \approx 23 \mathrm{~K}$. Upon approaching $T_{N 1}=2.3 \mathrm{~K}$, a magnetic modulation shows up which is incommensurate with the crystal structure. The periodicity reveals a weak temperature dependence and below $T_{N 2}$ $=1.9 \mathrm{~K}$ a locked-in commensurate magnetic structure with wave vector $\mathbf{q}=(3 / 4,3 / 4,0)$ is established. The incommensurate magnetic structure shows a deviation $\delta$ of the propagation vector from the commensurate values of approximately $\delta \approx 0.04$. Both the incommensurate, as well as the low-temperature commensurate magnetic structure with wave vector $\mathbf{q}=\left(q_{x}, q_{x}, 0\right), q_{x}=q_{y} \approx 0.75$ violate the symmetry of the cubic spinel structure. This may be an indication of an additional structural modulation, unresolved in the present neutron powder diffraction experiments. The broad magnetic diffraction intensity mainly originates from inelastic scattering processes due to magnetic excitations, but any deviation of the propagation vector from $(3 / 4,3 / 4,0)$ additionally contributes in the elastic channel.

\section{SUMMARY AND CONCLUSIONS}

We have presented results of a detailed neutron scattering investigation of the frustrated magnet $\mathrm{MnSc}_{2} \mathrm{~S}_{4}$. Neutron powder diffraction revealed a spiral magnetic structure characterized by a propagation vector of $\mathbf{q}=(3 / 4,3 / 4,0)$ and an ordered magnetic moment of $\mu_{\text {ord }}=4.05 \mu_{B}$ at $1.5 \mathrm{~K}$. Due to the low ordering temperature of $T_{N 1}=2.3 \mathrm{~K}$ saturation with the full magnetic moment of $5 \mu_{B}$ could not be achieved but requires considerable lower temperatures. Based on symme- 
try, the magnetic structure is incompatible with the cubic spinel type crystal structure. One possibility is that the crystal structure is in fact reduced but without affecting the powder diffraction pattern which reflects the average global structure. This would explain the double peak structure of the heat capacity and susceptibility in terms of a magnetically and structurally driven phase transition by a strong coupling of the spin and lattice degrees of freedom as, i.e., theoretically described by a spin-driven Jahn-Teller effect. ${ }^{12}$ The magnetic excitation spectra show a quasielastic response with a width of the order of $1 \mathrm{meV}$ corresponding to the magnetic energy scale. Upon approaching long-range magnetic order, a change from a quasielastic to an inelastic magnetic response is observed. In the magnetically ordered lowtemperature state well-defined magnon branches can be observed which have been fitted by an antiferromagnetic spin wave dispersion relation. Constant energy strips of $S(Q, \omega)$ with various energy width reveal the essentially inelastic origin of the additional broad diffraction component around the sharp, resolution limited magnetic Bragg reflections. Whereas strong magnetic correlations start to develop below $\left|\theta_{\mathrm{CW}}\right| \approx 23 \mathrm{~K}$, long-range magnetic order is consider- ably suppressed down to $T_{N} \approx\left|\theta_{\mathrm{CW}}\right| / 10=2.3 \mathrm{~K}$. The detailed magnetic ordering process is characterized by an incommensurate magnetic modulation around $T_{N 1}=2.3 \mathrm{~K}$ and shifts on cooling, becoming a locked-in commensurate structure below $T_{N 2}=1.9 \mathrm{~K}$. The frustrated magnet $\mathrm{MnSc}_{2} \mathrm{~S}_{4}$ therefore shows a transition from a high-temperature paramagnet $(T$ $\left.\geqslant \theta_{\mathrm{CW}}\right)$ via a spin-liquid phase for intermediate temperatures $\left(T_{N} \leqslant T \leqslant \theta_{\mathrm{CW}}\right)$ to a magnetically long-range ordered spin solid ground state for low temperatures $\left(T \leqslant T_{N}\right)$.

\section{ACKNOWLEDGMENTS}

This work was supported by the German Bundesministerium für Bildung und Forschung under Contract No. VDI/ EKM 13 N6917 and by the Deutsche Forschungsgemeinschaft DFG via SFB 484, Augsburg. We thank J. Hemberger for providing Fig. 1 and T. Hansen for allocating test beam time on D20. Help during the refinement of the magnetic structure by M. Reehuis and fruitful discussions with C. Vettier are gratefully acknowledged.
*Electronic address: Alexander.Krimmel@physik.uni-augsburg.de

${ }^{1}$ A. P. Ramirez, in Handbook of Magnetic Materials, edited by K. H. J. Buschow (Elsevier Science, Amsterdam 2001), Vol. 13, p. 423.

${ }^{2}$ B. Canals and C. Lacroix, Phys. Rev. Lett. 80, 2933 (1998).

${ }^{3}$ A. P. Ramirez, A. Hayashi, R. J. Cava, R. Siddhartan, and B. S. Shastry, Nature (London) 399, 333 (1999).

${ }^{4}$ S. P. Bramwell and M. J. P. Gringas, Science 249, 1495 (2001).

${ }^{5}$ A. J. Garcia-Adeva and D. L. Huber, Phys. Rev. Lett. 85, 4598 (2000).

${ }^{6}$ P. G. Radaelli, Y. Horibe, M. J. Gutmann, H. Ishibashi, C. H. Chen, R. M. Ibberson, Y. Koyama, Y.-S. Hor, V. Kiryukhin, and S.-W. Cheong, Nature (London) 416, 155 (2002).

${ }^{7}$ S.-H. Lee, C. Broholm, W. Ratcliff, G. Gasparovic, Q. Huang, T. H. Kim, and S.-W. Cheong, Nature (London) 418, 856 (2002).

${ }^{8}$ A. Krimmel, A. Loidl, M. Klemm, S. Horn, and H. Schober, Phys. Rev. Lett. 82, 2919 (1999).

${ }^{9}$ E. Berg, E. Altman, and A. Auerbach, Phys. Rev. Lett. 90, 147204 (2003).

${ }^{10}$ M. Reehuis, A. Krimmel, N. Büttgen, A. Loidl, and A. Prokofiev, Eur. Phys. J. B 35, 311 (2003).

${ }^{11}$ O. Tchernyshyov, R. Moessner, and S. L. Sondhi, Phys. Rev. Lett.
88, 067203 (2002).

${ }^{12}$ Y. Yamashita and K. Ueda, Phys. Rev. Lett. 85, 4960 (2000).

${ }^{13}$ A. Krimmel, M. Mücksch, V. Tsurkan, M. M. Koza, H. Mutka, and A. Loidl, Phys. Rev. Lett. 94, 237402 (2005).

${ }^{14}$ R. Fichtl, V. Tsurkan, P. Lunkenheimer, J. Hemberger, V. Fritsch, H.-A. Krug von Nidda, E.-W. Scheidt, and A. Loidl, Phys. Rev. Lett. 94, 027601 (2005).

${ }^{15}$ J. Hemberger, P. Lunkenheimer, R. Fichtl, H.-A. Krug von Nidda, V. Tsurkan, and A. Loidl, Nature (London) 434, 364 (2005).

${ }^{16}$ V. Fritsch, J. Hemberger, N. Büttgen, E.-W. Scheidt, H.-A. Krug von Nidda, A. Loidl, and V. Tsurkan, Phys. Rev. Lett. 92, 116401 (2004).

${ }^{17}$ N. Büttgen, J. Hemberger, V. Fritsch, A. Krimmel, M. Mücksch, H.-A. Krug von Nidda, P. Lunkenheimer, R. Fichtl, V. Tsurkan, and A. Loidl, New J. Phys. 6, 191 (2004).

${ }^{18}$ W. L. Roth, J. Phys. (Paris) 25, 507 (1964).

${ }^{19}$ S. Reil, H.-J. Stork, and H. Haeseler, J. Alloys Compd. 334, 92 (2002).

${ }^{20}$ J. Rodriguez-Carvajal, Physica B 192, 55 (1993).

${ }^{21}$ S. Giri, H. Nakamura, and T. Kohara, Phys. Rev. B 72, 132404 (2005).

${ }^{22} \mathrm{http} / / / \mathrm{www}$. ill.fr/data_treatment/lamp/front.html 